\title{
Condromatose sinovial: caso raro em articulação temporomandibular
}

\author{
Synovial chondromatosis: a rare case in temporomandibular joint
}

\section{Condromatosis sinovial: caso raro en la articulación temporomandibular}

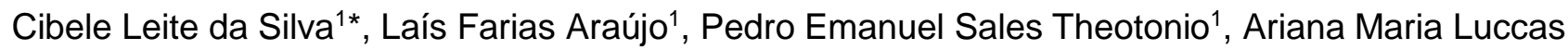
Costa Loureiro², Rafaella Amorim Bittencourt Maranhão de Araújo², Luciano Schwartz Lessa Filho, Pedro Thalles Bernardo de Carvalho Nogueira ${ }^{1}$.

\section{RESUMO}

Objetivo: Relatar um caso cínico de condromatose sinovial em articulação temporomandibular direita. Detalhamento do caso: Durante o exame físico foi observado um aumento de volume na região pré-auricular direita, assimetria facial e limitação de abertura bucal, além de deflexão durante a abertura. No exame de imagem (tomografia computadorizada) observou-se imagem hiperdensa em região de articulação temporomandibular direita, recobrindo todo o côndilo mandibular direito. Logo, foi realizada uma biópsia incisional da região, e encaminhada para analise histopatológica onde foi obtido o diagnóstico de condromatose sinovial. Como tratamento definitivo, foi realizada a exérese total da lesão sob anestesia geral, com o acesso pré-auricular. Atualmente, a paciente encontra-se em pós-operatório de dois anos, apresenta abertura de boca satisfatória, ausência de queixas álgicas e boa abertura bucal. No exame tomográfico de controle não foi observado qualquer sinal de recidiva. Considerações finais: $O$ diagnóstico de condromatose sinovial na articulação temporomandibular é desafiador. Caracteriza-se por ser uma lesão rara e comumente descartada do diagnóstico diferencial inicial. Desta forma, é relevante a realização de exames radiológicos e histopatológicos para auxilio no diagnóstico correto.

Palavras-chave: Transtornos da articulação temporomandibular, Condromatose sinovial, Neoplasias.

\section{ABSTRACT}

Objective: The current study aimed at reporting a clinical case of synovial chondromatosis in the right temporomandibular joint. Details of the case: When the physical exam was carried out, a volume increase in the right preauricular region, facial asymmetry and limited buccal opening were noticed; as well as deflection during the opening. The imaging exam (computerized tomography) showed a hyper dense image in the right temporomandibular joint region, covering all the right mandibular condyle. Therefore, an incisional biopsy of the region was accomplished and sent for a histopathological analysis, where the synovial chondromatosis diagnosis was made. As a final treatment, the total exeresis of the lesion was carried out under general anesthesia, with preauricular access. Currently, the patient is in the second postoperative year showing a satisfactory mouth opening and good buccal opening. The control tomographic exam showed no sign of remission. Final considerations: The diagnosis of synovial chondromatosis in the temporomandibular joint is challenging. It is characterized by being a rare and commonly ruled out lesion from the initial differential diagnosis. Thus, it is relevant to perform radiological and histopathological exams to aid in the correct diagnosis.

Keywords: Temporomandibular joint disorders, Chondromatosis synovial, Neoplasms.

\section{RESUMEN}

Objetivo: El objetivo de este estudio era informar un caso clínico de condromatosis sinovial en articulación mandibular derecha. Detalles del caso: Durante el examen físico se observó un aumento de volumen en la región pre-auricular derecha, asimetría facial y limitación de apertura bucal, además de deflexión durante la apertura. En el examen de imagen (tomografía computarizada) se observó imagen hiperdensa en región de ATM derecha, cubriendo todo el cóndilo mandibular derecho. Luego, se realizó una biopsia incisional de la región, y se encaminó al análisis histopatológico donde se obtuvo el diagnóstico de condromatosis sinovial. Como tratamiento definitivo, se realizó la exéresis total de la lesión bajo anestesia general, con el acceso pre-auricular. Actualmente, la paciente se encuentra en postoperatorio de dos años, presenta apertura de boca satisfactoria y ausencia de quejas álgicas. En el examen tomográfico de control no se observó ningún signo de reincidencia. Consideraciones finales: El diagnóstico de condromatosis sinovial en la articulación temporomandibular es desafiante. Se caracteriza por ser una lesión rara y comúnmente descartada del diagnóstico diferencial inicial. De esta forma, es relevante la realización de exámenes radiológicos y histopatológicos para ayudar en el diagnóstico correcto.

Palabras claves: Trastornos de la articulación temporomandibular, Condromatosis sinovial, Neoplasias.

${ }^{1}$ Centro Universitário Tiradentes, Maceió - AL. *E- mail: cibeleleleite8@hotmail.com

${ }^{2}$ Centro Universitário Cesmac, Maceió - AL. 


\section{INTRODUÇÃO}

A condromatose sinovial se caracteriza como um crescimento neoplásico benigno, ou não neoplásico, que afeta a articulação do tipo diartrose (KAPANDJI Al, 2008). Comumente se apresentam como nódulos metaplásicos cartilaginosos, de diferentes tamanhos, podendo ser únicos ou destacados como corpos soltos, dentro do tecido conjuntivo sinovial (HOLMLUND AB, et al., 2003). Normalmente é limitado ao espaço articular e incide mais comumente joelho, quadril, cotovelo e ombro. Ocorrendo raramente na articulação temporomandibular (HOLMLUND AB, et al., 2003; BONATTI BS, et al., 2008; GOIZUETA-ADAME CC e GONZALEZ-GARCIA R, 2010; REINISH El, et al., 1997; SATO J, et al., 2002).

Os sintomas mais comuns são: dor localizada ou referida, crepitação, má oclusão, desvio e diminuição da amplitude mandibular (IVASK O, et al., 2015; SINK J, et al., 2014). Entretanto, estes sintomas também são comumente encontrados em diversos problemas articulares, o que torna o diagnostico complexo e muitas vezes realizado de forma tardia (ALMEIDA LE, et al., 2002). Para auxiliar o diagnóstico de condromatose sinovial é imprescindível a averiguação de exames complementares, como tomografia computadorizada (TC) e ressonância magnética (RMN) para comparar com outras desordens, e analisar das características da lesão, afim de elucidar para o correto diagnóstico (HOLMLUND AB, et al., 2003; BONATTI BS, et al., 2008; BENSLAMA L, et al., 2018; LIU X, et al., 2016).

A etiopatogenia é desconhecida, no entanto Mcain JP e Rua HD (1989) dividiram a condromatose sinovial em duas categorias, a primaria advinda da não identificação de fatores etiológicos, onde existe a formação de nódulos de cartilagem metaplásicas dos restos mesenquimais, com ou sem calcificação (cartilaginosos ou osteocartilaginosos) onde os corpos cartilaginosos ficam confinados no espaço articular superior (HOLMLUND AB, et al., 2003; NORMAN JE, et al., 1988). Já os secundários, provenientes do trauma facial, podem causar o desalojamento de tecido cartilaginoso ou ósseo no espaço articular, onde alojam-se no tecido sinovial, fazendo com que os fragmentos sejam cobertos por uma camada de membrana sinovial, que lhes proporciona nutrição (REINISH El, et al., 1997; BENSLAMA L, et al., 2018; LIU X, et al., 2016).

O tratamento recomendado para a condromatose sinovial é a remoção completa dos corpos soltos e é imprescindível para sucesso do tratamento, e para diminuir as taxas de recorrência e consequentemente de malignização (HOLMLUND AB, et al., 2003; DAVIS RI, et al., 1998). A técnica mais conservadora e menos invasiva é a artroscopia, que é observada na literatura, entretanto possui um limite de acordo com o tamanho da lesão (CARLS FR, et al., 1995). A técnica mais convencional e recomendada é a aberta, realizada pela técnica pré-auricular, onde irá ter acesso direto à cápsula articular, proporcionando uma enucleação adequada (HOLMLUND AB, et al., 2003; GOIZUETA-ADAME CC e GONZALEZ-GARCIA R, 2010). Discectomia, Condilectomia e Sinovectomia também são relatados na literatura, entretanto são tratamentos para casos mais avançados, em que haja lesões as estruturas nobres condizentes com 0 a estrutura anatômica (HOLMLUND AB, et al., 2003).

O objetivo do presente artigo é relatar um caso cínico de Condromatose Sinovial em articulação temporomandibular direita e fornecer informações acerca de seu diagnóstico e tratamento.

\section{DETALHAMENTO DE CASO}

Paciente GSM, gênero feminino, 50 anos de idade, comparece ao serviço de um hospital em Maceió e durante a anamnese relatou dificuldade em abrir a boca e sintomatologia dolorosa no lado direito da face com evolução de aproximadamente 10 anos. Ao exame físico, foi observado discreto aumento de volume em região pré-auricular direita, assimetria facial caracterizada por laterognatismo e limitação de abertura bucal $(30 \mathrm{~mm})$, além de deflexão mandibular durante a abertura bucal. A paciente negava deformidades congênitas, trauma ou realização de cirurgias prévias na face.

Ao exame de imagem (tomografia computadorizada 3D) observou-se imagem hiperdensa em região de ATM direita, iniciando no arco zigomático e recobrindo todo o côndilo mandibular, aparentemente alterando a forma da articulação (Figura 1). Diante disso, foi realizada uma biópsia incisional da região, e a peça encaminhada para análise histopatológica onde confirmou-se o diagnóstico de Condromatose Sinovial. 
Figura 1 - Lesão patológica (Condromatose Sinovial) - Tomografia computadorizada.

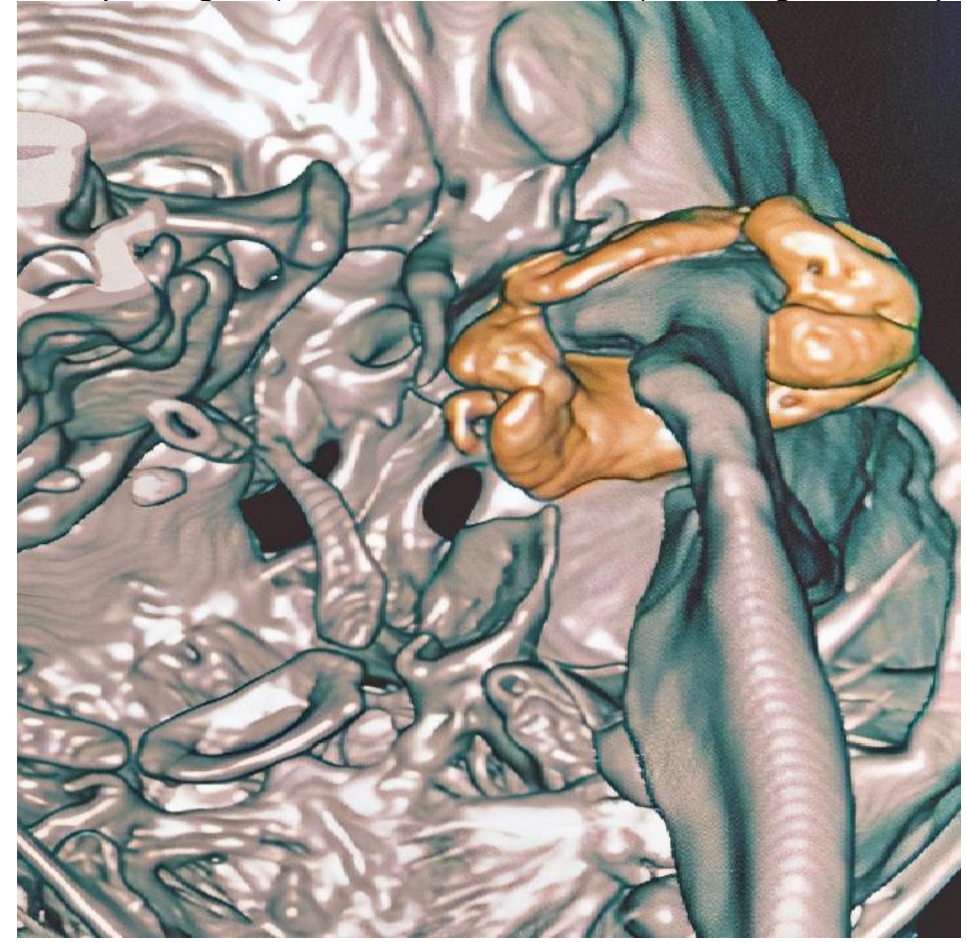

Fonte: Silva CL, et al., 2021.

Como tratamento definitivo, possibilitado pelo acesso pré-auricular direito (Figura 2) foi realizado exérese total da lesão que se inciava no arco zigomático e circundava a região de côndilo mandibular, sob anestesia geral, em centro cirúrgico, preservando todas as estruturas articulares e restabelecendo o funcionamento normal da mandíbula (Figura 3). Vale ressaltar que a paciente optou por não tratar a assimetria através de cirurgia ortognática por questões pessoais.

Figura 2 - Acesso pré-auricular realizado e identificação da lesão patológica.

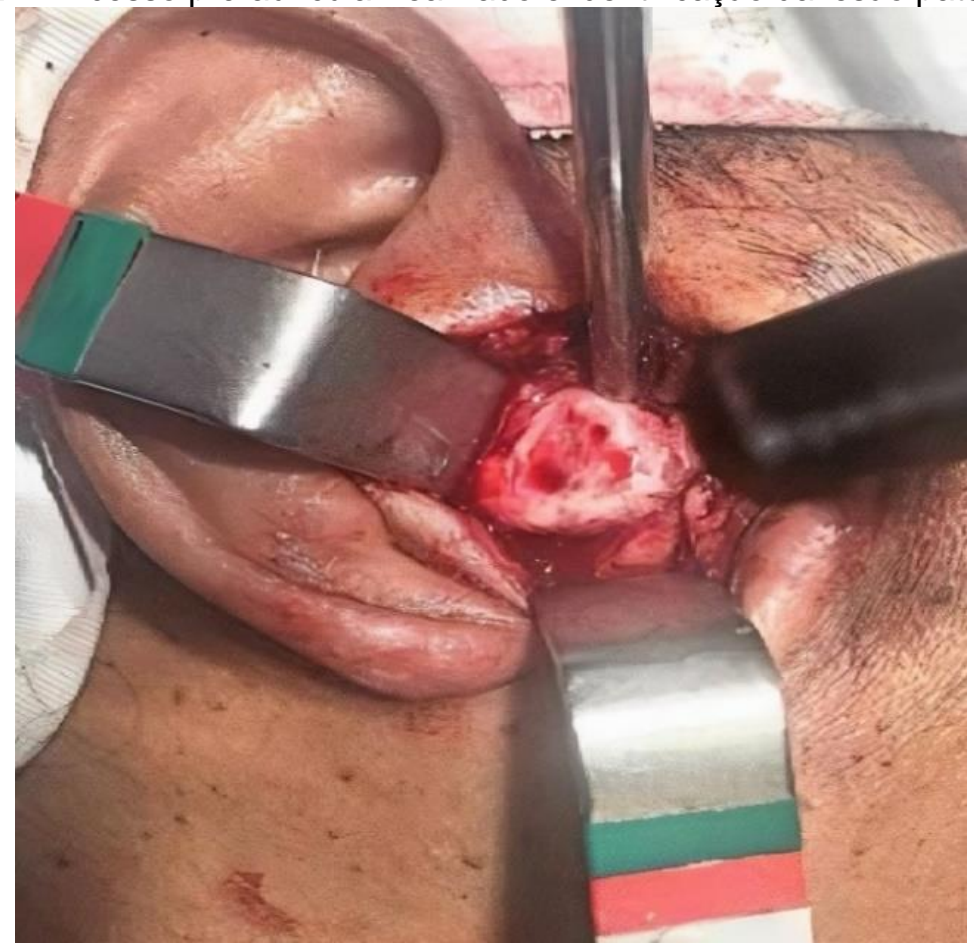

Fonte: Silva CL, et al., 2021. 
Figura 3 - Exérese total da lesão.

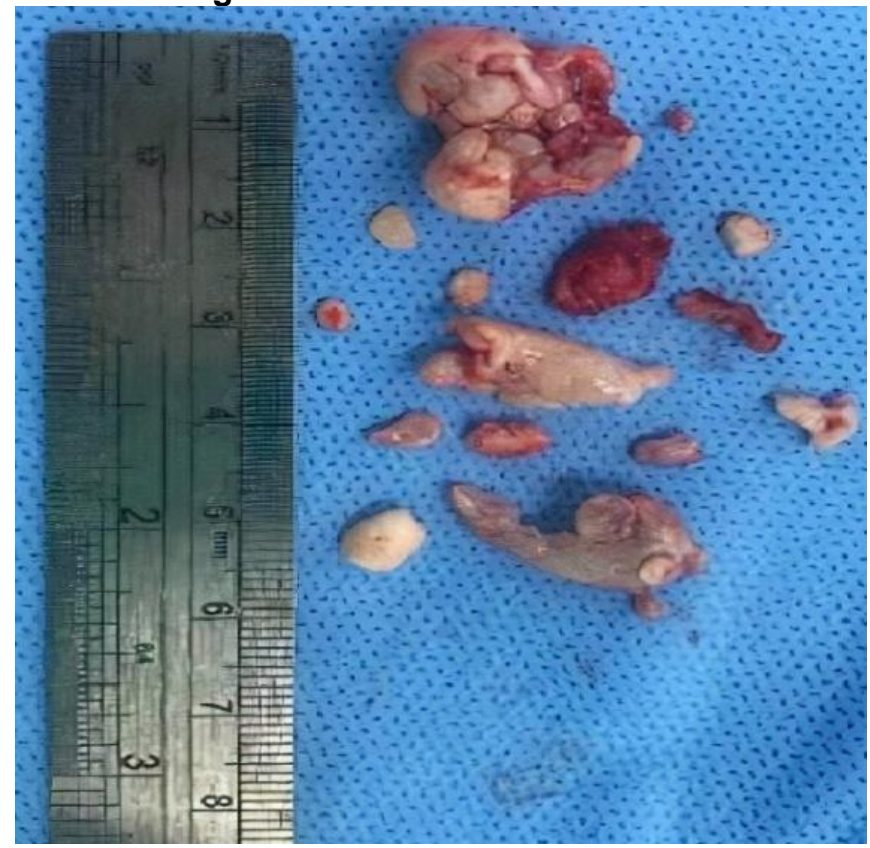

Fonte: Silva CL, et al., 2021.

No período pós-operatório a paciente foi medicada com antibiótico, anti-inflamatório e analgésico em um período de cinco dias e submetida à reabilitação precoce através de fisioterapia para minimizar os riscos de anquilose e possibilitar um quadro mais favorável. Não foram observados sinais de infecção ou inflamação no período pós-operatório. Desta forma, a paciente foi instruída para retorno de avaliação em tempos periódicos.

Atualmente, a paciente encontra-se em pós-operatório de dois anos, apresentando boa abertura bucal $(45 \mathrm{~mm})$, ausência de queixas álgicas e sem alterações motoras na musculatura da face. No exame tomográfico de controle não foi observado qualquer sinal de recidiva e preservação das estruturas ósseas (Figura 4). A paciente foi orientada a retornar em caso de sintomatologia dolorosa.

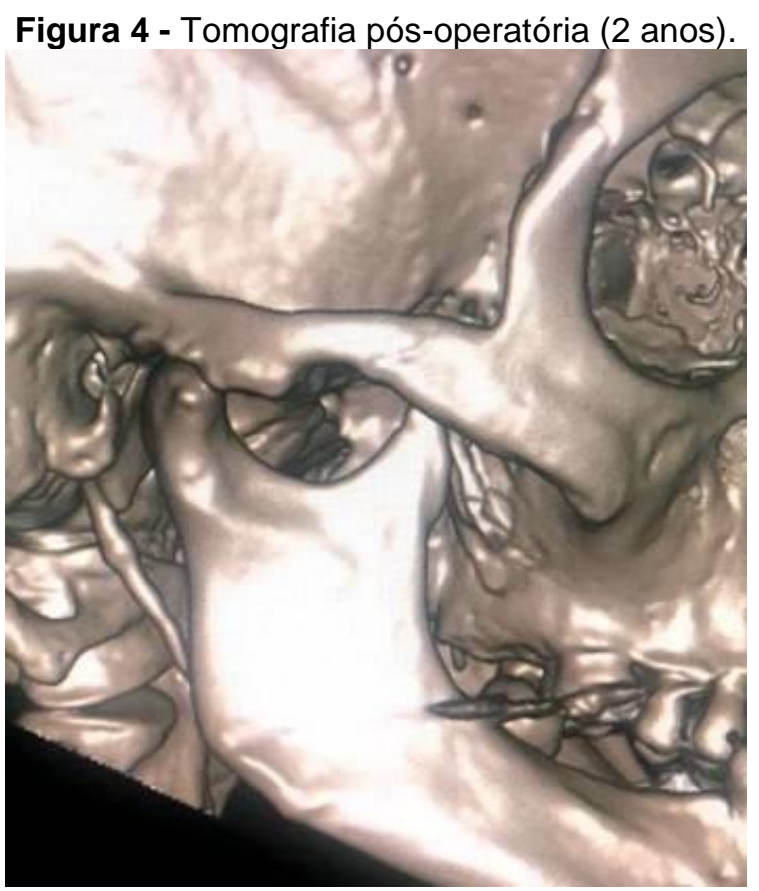

Fonte: Silva CL, et al., 2021. 


\section{DISCUSSÃO}

A condromatose sinovial (CS) é uma condição benigna incomum, e geralmente tem um crescimento lento. Raramente acomete a articulação temporomandibular, visto que incide principalmente em articulações maiores, como ombro, cotovelo, quadril e joelho (HOLMLUND AB, et al., 2003; BALASUDARAM A, et al., 2009). No estudo de Shah SB, et al. (2011) foram relatados 241 casos de condromatose sinovial da articulação temporomandibular. Sendo estes casos de 1764 até 2010.

Gonzalez-Perez LM, et al. (2011) aponta que na Condromatose Sinovial existe uma metaplasia progressiva cartilaginosa dos restos mesenquimais do tecido sinovial, onde os corpos cartilaginosos formados, que podem ser únicos e mais robustos ou destacados como corpos soltos, apresentando-se com ou sem calcificação (cartilaginosos ou osteocartilaginosos) e geralmente ficam localizados no espaço articular superior (YOKOTA N, et al., 2008). Em lesões mais agressivas pode-se notar a reabsorção de estruturas importantes como o osso subjacente ou a capsula articular, chegando ou não a área peri-radicular, resultando em um prognóstico desfavorável (PAU M, et al., 2014).

O presente estudo trata-se de uma paciente do sexo feminino, com Condromatose Sinovial, corroborando com as literaturas descritas, entretanto na articulação temporomandibular, que enquanto região acometida tem rara incidência. Observou-se que as lesões cartilaginosas se encontravam como um grande corpo ao redor da articulação temporomandibular e diversos corpos soltos destacados no espaço articular superior.

A etiologia é controversa, e ainda muito pouco conhecida, Holmlund AB, et.al., (2003) por sua vez, destaca duas patogêneses relevantes, a tumefação advinda de processo neoplásico e a metaplasia dos tecidos embrionários. Lustmann $\mathrm{J}$ e Zeltser $\mathrm{R}$, (1989) dividiu a etiologia da condromatose sinovial em duas segmentações, sendo a primária, de origem desconhecida, porém outros autores acreditam que a mesma é advinda de remanescentes de tecido sinovial que sofreram metaplasia (DASPIT P e SPETZLER RF, 1989). E a secundaria, por sua vez, tem origem de traumas, ou microtraumas repetitivos, infecções, ou doenças articulares. Acredita-se que a origem da lesão relatada neste artigo, seja primaria, partindo do pressuposto onde a mesma relatou não ter sofrido traumas, infecções ou doenças articulares pré-existentes.

Em 1980 foi adotado o termo condromatose sinovial (NORMAN JE, et al., 1988). A lesão tem predileção pelo sexo feminino em aproximadamente 2:1. O lado direito é afetado com maior frequência em cerca de 1:1,2 dos pacientes. Normalmente só uma das ATM é afetada (PENG LW, et al., 2009). Na maioria dos casos o diagnóstico é tardio, e varia dos 39 a 55,4 anos de idade (MARTIN-GRANIZO R, et al., 2005; TESTAVERDE L, et al., 2011; VON LINDERN JJ, et al., 2002).

Em alguns casos, foi verificado vertigem, zumbido, perda auditiva e/ou sintomas neurológicos (NORMAN JE, et al., 1988), além dos sinais e sintomas comumente reconhecidos, como: dor localizada ou referida, crepitação, má oclusão, desvio e diminuição da amplitude mandibular, que são também associados a outras desordens temporomandibulares (IVASK O, et al., 2015; SINK J, et al., 2014). No entanto existe um sinal mais especifico que foi encontrado majoritariamente nos casos documentados, que é o inchaço na região préauricular com ausência de queixa acidental, aparente, do paciente (GUIJARRO-MARTINEZ R, et al., 2011). A taxa de recidiva é rara (HOLMLUND AB, et al., 2003). Shah SB, et.al. (2011) relataram 4 casos de recidiva de 1764 até 2010 .

No presente estudo, a predileção pelo sexo feminino, faixa etária e pela articulação temporomandibular direita vista em outros artigos foi confirmado. Assim como os sinais e sintomas mais comuns. Neste caso também foi avaliado e confirmado o sinal mais específico da condromatose sinovial, o aumento de volume na região pré-auricular não vinculado a trauma. O monitoramento clínico pós operatório da paciente deste estudo foi de 2 anos, sem sinal de recidiva, sinais ou queixas algicas, não sendo necessario, desta forma, outro acompanhamento caso a paciente continue sem sintomatologias ou sinais que sugiram recidiva.

A transformação maligna da condromatose sinovial é incomum. Os casos em que houve recidiva da lesão apresentaram-se como os mais propensos a essa transformação (MULLINS F, et al., 1965). Davis RI, et al. (1998) relataram que 3 dos 9 pacientes que tiveram recidiva da condromatose sinovial, apresentaram 
transformação para condrossarcoma no joelho. Entretanto, Shah SB, et al. (2011) no relato de seus 241 casos não relatou condrossarcoma como transformação maligna em articulação temporomandibular. Estudos recentes também não mostram esta transformação (BENSLAMA L, et al., 2018; PAU M, et al., 2014). Entretanto, Mullins F, et al. (1965) publicou um caso, com dados clínicos e radiográficos, sobre a transformação de condromatose sinovial em condrossarcoma. É válido salientar que a literatura é escassa a respeito da transformação neoplásica da articulação temporomandibular. No caso relatado deste artigo, não foi encontrado sinais de malignização.

Para o diagnóstico é imprescindível o exame de imagens, para que sua localização e extensão possam ser visualizadas, possibilitando um planejamento cirúrgico adequado. As radiografias podem identificar corpos livres quando eles se encontram calcificados em apenas duas dimensões ((HOLMLUND AB, et al., 2003), porém fornecem informações muito limitadas quando os nódulos se encontram nas fases iniciais (HOLMLUND AB, et al., 2003; PEYROT H, et al., 2014). Balasudaram A, et al. (2009) aponta que no exame radiográfico é possível identificar um alargamento do espaço articular, degeneração das superfícies articulares, a expansão da capsula articular e a presença de corpos livres.

A tomografia e a Ressonância magnética são as imagens mais úteis para o diagnóstico da patologia (HOLMLUND AB, et al., 2003; REINISH El, et al., 1997; DASPIT P e SPETZLER RF, 1989; XU WH, et al., 2007; VAN INGEN JM, et al., 1990). A tomografia ajuda a identificar corpos livres, erosões da fossa mandibular, alterações da base do crânio, cabeça do côndilo e extensões intracranianas, mostra também o tamanho e localização dos corpos calcificados além de seu grau de calcificação (HOLMLUND AB, et al., 2003; VAN INGEN JM, et al., 1990; ARDEKIAN L, et al., 2005).

A ressonância magnética $(\mathrm{RM})$ é o padrão ouro no diagnóstico das condromatoses sinoviais, e possibilita visualizar os corpos soltos no fluido sinovial (HOLMLUND AB, et al., 2003; PEYROT H, et al., 2014; XU WH, et al., 2007). É possível a identificação dos nódulos antes de sua ossificação, ou seja, em sua fase inicial, fazendo com que promova a visualização e identificação com estruturas importantes, sua extensão e também se a dura-mater está envolvida (HOLMLUND AB, et al., 2003; PAU M, et al., 2014; ARDEKIAN L, et al., 2005; ACAR GO, et al., 2007; WANG P, et al., 2012).

A RM também pode detectar o edema articular e o limite da lesão, permitindo a exclusão da possibilidade de um tumor (HOLMLUND AB, et al., 2003) Acar GO, et al. (2007) também notou que a ressonância é eficaz nas fases inicias da desordem.

Nos casos do estudo de GUARDA-NARDINI L, et al. (2010) a tomografia computadorizada e a ressonância magnética foram notadas como as mais realizadas seguido por ordem decrescente de utilização, da ortopantomografia, mais conhecida como radiografia panorâmica, artrografia, tomografia volumétrica cone beam e tomografia sagital. Em contrapartida, mesmo com uma significativa melhora nas qualidades dos instrumentos utilizados de exame de imagem para diagnosticar essa patologia (DAVIS RI, et al., 1998), os corpos livres ainda são poucos identificados a exames imagilongicos (HOLMLUND AB, et al., 2003).

Há também uma linha de pensamento, onde a combinação de RM, TC, CBCT, e outras técnicas são melhores no diagnóstico e possivelmente tratamento do caso (LIU X, et al., 2016). A artroscopia é também utilizada para diagnóstico desta desordem proliferativa (HOLMLUND AB, et al., 2003).

A ressonância magnética constitui um padrão ouro para esta patologia, seguido da tomografia computadorizada. Devido questões monetárias, no caso descrito foi realizado somente a tomografia computadorizada, onde foi possível a avaliação completa da articulação temporomandibular e patologia, sem prejuízo para o diagnóstico e possibilitando um tratamento efetivo.

O diagnóstico de condromatose sinovial na articulação temporomandibular é desafiador, visto que os sinais e sintomas são semelhantes a outras desordens articulares. Além disso, por constituir uma lesão rara é comumente descartada do diagnóstico diferencial inicial. Desta forma, é relevante a realização de uma anamnese detalhada e a associação de exames radiológicos e histopatológicos de boa qualidade para auxilio no diagnóstico correto. 


\section{REFERÊNCIAS}

1. ACAR GO, et al. Synovial chondromatosis of the temporomandibular joint with skull base extension. J Craniofac surg. 2007; 1: 241-243.

2. ALMEIDA LE, et al. Condromatose Sinovial da articulação têmporo-mandibular - Relato de um caso. Revista ABO Nac. 2002; 5: 305-308.

3. ARDEKIAN L, et al. Synovial chondromatosis of the temporomandibular joint: Report and Analysis of eleven cases. Journal Oral Maxillofac Surg. 2005; 7:941-7.

4. BALASUDARAM A, et al. Radiographic diagnosis of synovial chondromatosis of the temporomandibular joint: a case report. J Can Dent Assoc. 2009; 75:711-714.

5. BENSLAMA L, et al. Synovial chondromatosis of the temporomandibular joint: Report and analysis of $12 \mathrm{cases}$. J Stomatol Oral Maxillofac Surg. 2018; 120: 476-479.

6. BONATTI BS, et al. Condromatose sinovial de articulação têmporo-mandibular. Rev Bras Otorrinolaringol. $2008 ; 3: 480$.

7. CARLS FR, et al. Loose Bofird in the temporomandibular joint: the advantages of arthroscopy. J Craniomaxillofac Surg. 1995; 23:215-221.

8. DASPIT P, SPETZLER RF. Synovial Chondromatosis of the temporomandibular joint with intracranial extension: case report. J Neurosurg. 1989; 70: 121-123

9. DAVIS RI, et al. Primary Synovial Chondromatosis: a clinicopathologic review and assessment of malignant potential. Hum Pathol. 1998; 29: 683-688.

10. GOIZUETA-ADAME CC, GONZÁLEZ-GARCÍA R. Synovial Chondromatosis of the temporomandibular joint: reporto f 2 patients whose joints were reconstructed with costochondral graft and alloplastic prosthesis. British Journal of Oral and Maxillofacial Surgery. 2010; 48: 374-377.

11. GONZALEZ-PEREZ LM, et al. Temporomandibular joint synovial chondromatosis with a traumatic etiology. Int $\mathrm{J}$ Oral Maxillofac Surg. 2011; 40: 330-334.

12. GUARDA-NARDINI L, et al. Synovial Chonromatosis of the temporomandibular joint: a case description with systematic literature review. J. Oral Maxillofac. Sug., 2010; 39: 745-755.

13. GUIJARRO-MARTINEZ R, et al. Bilateral synovial chondromatosis of the temporomandibular joint. Journal Maxillofac Surg. 2011; 261.

14. HOLMLUND AB, et al. Synovial Chondromatosis of the temporomandibular Joint. Clinical, surgical and histological aspects. J Oral Maxilofac Surg., 2003; 32;143-147.

15. IVASK O, et al. Synovial Chondromatosis in the temporomandibular joint: case report with review of the literature. Stomatologija, Baltic Dental and Maxillofacial Journal, 2015; 17: 97-101.

16. KAPANDJI AI. Fisiologia articular. Grupo Editora Nacional. 2008; 6: 3.

17. LIU X, et al. Clinical and Imaging Findings of Temporomandibular Joint Synovial Chondromatosis: An Analysis of 10 cases and literature review. J Oral Maxillofac Surg. 2016; 11: 2159-2168.

18. LUSTMANN J, ZELTSER R. Synovial chondromatosis of the temporomandibular joint. Review of the literature and case report. Int Journal Oral Maxillofac Surg., 1989; 2: 90-94.

19. MARTIN-GRANIZO R, et al. Synovial chondromatosis of the temporomandibular joint: a clinical, radiological and histological study. Med Oral Patol Oral Cir Bucal, 2005; 10: 272-276.

20. REINISH El, et al. Primary synovial chondromatosis of the temporomandibular joint whith suspected traumatic etiology: report of a case. Int J Oral Maxillofac Surg. 1997; 26: 419-422.

21. MCAIN JP, RUA HD. Arthroscopic observation and treatment of synovial chondromatosis of the temporomandibular joint. Report of a case and review of the literature. Int J Oral Maxillofac Surg. 1989; 18:233-236.

22. MULLINS F, et al. Chondrosarcoma following synovial chondromatosis: a case study. Cancer. 1965; $18: 1180$.

23. NORMAN JE, et al. Synovial osteochondromatosis of the temporomandibular joint. An historical review with presentation of 3 cases. Journal Craniomaxillofac Surg. 1988; 16: 212-220.

24. PAU M, et al. Surgical treatment of synovial chondromatosis of the temporomandibular joint whit erosion of the skull base: a case report and review of the literature. Int J Oral Maxillofac Surg. 2014; 43: 600-605.

25. PENG LW, et al. Synovial chondromatosis of the temporomandibular joint: a case report with bilateral occurrence. J Oral Maxillofac Surg. 2009; 67: 893-895.

26. PEYROT $\mathrm{H}$, et al. Synovial chondromatosis of the temporomandibular joint: CT and MRI findings. Diagn Interv Imaging. 2014; 95: 613-614.

27. SATO J, et al. The expression of fibroblast growth fator -2 and fibroblast growth fator receptor- 1 in chondrocytes in synovial chondromatosis of the temporomandibular joint. Report of two cases. J Oral Maxillofac Surg. 2002; 31: 532536.

28. SHAH SB, et al. Synovial Chondromatosis of temporomandibular Joint: Journey through 25 decades and a case report. J Oral Maxillofac Surg. 2011; 69: 2795-2814.

29. SINK J, et al. Synovial Chondromatosis of the temporomandibular joint: Clinical, cytologic, histologic, radiologic, therapeutic aspects, and differential diagnosis of an uncommon lesion. Oral Surg Oral Med Oral Pathol Oral Radio. 2014; 3: 269.

30. TESTAVERDE L, et al. MR finding in synovial chondromatosis of the temporo-mandibular joint: our experience and review of literature. Eur J Radiol. 2011; 78: 414-418.

31. VAN INGEN JM, et al. CT dignosis of synovial chondromatosis of the temporomandibular joint. BR J Oral Maxilofac Surg., 1990; 28: 164-167.

32. VON LINDERN JJ, et al. Synovial chondromatosis of the temporomandibular joint: clinical, diagnostic, and histomorphologic findings. Oral Surg Oral Med Oral Pathol Oral Radiol Endod. 2002; 94: 31-38.

33. WANG P, et al. Synovial chondromatosis of the temporomandibular joint: MRI findings with pathological comparison. Dentomaxillofac Radiol. 2012; 41: 110-116.

34. $\mathrm{XU} \mathrm{WH}$, et al. Synovial Chondromatosis of the temporomandibular joint with middle cranial fossa extension. $\mathrm{J}$ oral Maxillofac Surg., 2007; 36: 652-655.

35. YOKOTA N, et al. Synovial chondromatosis of the temporomandibular joint with intracranial extension. Neurol Med Chir., 2008; 48: 266-270. 